\title{
Caracterización molecular de maíces locales de la reserva de la Biosfera La Sepultura, México ${ }^{1}$
}

\section{Molecular characterization of local maize varieties from the Biosphere Reserve La Sepultura, Mexico}

\author{
Manuel Antonio Hernández-Ramos ${ }^{2}$, Luis A. Rodríguez-Larramendi ${ }^{2}$,Francisco Guevara-Hernández ${ }^{3}$, \\ María de los Ángeles Rosales-Esquinca ${ }^{3}$, René Pinto-Ruiz, ${ }^{3}$ Rodobaldo Ortiz-Pérez ${ }^{4}$
}

\section{RESUMEN}

El objetivo de este trabajo fue la caracterización molecular y filogenética de poblaciones de maíces locales de la Reserva de la Biosfera La Sepultura (REBISE), México. Se colectaron diecinueve poblaciones de maíz en nueve comunidades de la REBISE durante el ciclo agrícola 20122013. Se incluyeron además, dos variedades comerciales (VS-536 y VS-424), un híbrido comercial (H-MX3), un maíz recientemente introducido (Costeño), dos materiales de las razas Cónico chalqueño y elotes cónicos, y un teocintle (Zea mays ssp. Mexicana). En total fueron 26 muestras diferentes. La caracterización se realizó mediante RCP con marcadores inter-microsatélites (ISSR). Se amplificaron 113 fragmentos, en un rango de 150 a 2200 pb de los cuales $85,8 \%$ fueron polimórficos. Las secuencias "AG", "AC" y "GAA" tuvieron mayor número de bandas amplificadas y mayor polimorfismo. Los cebadores UBC834, I9 y UBC868 fueron los que mejor amplificaron. Las similitudes dentro de las bandas polimórficas pueden deberse a su igualdad como variedades, razas, especie, o al efecto de su polinización cruzada. El dendograma obtenido mostró una correlación cofenética relativamente baja $(\mathrm{r}=0,721)$ con un grado de confiabilidad bajo; no obstante, se integran claramente cuatro grupos principales. El primero se denominó maíces

\begin{abstract}
The objective of this research was the molecular and phylogenetic characterization of local maize populations of the La Sepultura Biosphere Reserve (REBISE), Mexico. In nine communities from the REBISE, Mexico, nineteen populations of local maize were sampled during 2012-2013. Two improved commercial varieties (Vs-536 and V-424), a commercial hybrid (H-MX3), a local corn (Coastal) recently introduced in the REBISE, two local materials from the state of Tlaxcala, Mexico; both from Chalqueño race and conical Elotes (young corns) and a teosinte (Zea mays ssp. Mexicana) were included. They totaled twenty six different samples. The characterization was done with the PCR technique and inter-type microsatellite genetic markers. In total 113, fragments were amplified and that ranged from 150-2200 bp, of which $85,8 \%$ were polymorphic. Sequences "AG", "AC" and "GAA" showed a greater number of amplified bands and higher polymorphism. The primers comprised of UBC834, I9 and UBC868 amplified the best. The similarities found within the polymorphic bands may be due to their equality such as varieties, races, species or genetic combination effect, a result of their crosspollination. The dendrogram obtained showed a relatively low cophenetic correlation $(r=0,721)$, with a low degree
\end{abstract}

1 Recibido: 28 de octubre, 2015. Aceptado: 13 de junio, 2016. Este trabajo formó parte de la investigación realizada en la tesis de maestría del primer autor en Ciencias en Producción Agropecuaria Tropical, Universidad Autónoma de Chiapas, titulada: "Maíces locales con potencial de uso múltiple en un área natural protegida de Chiapas", en México, enmarcada en el proyecto "Caracterización de las estrategias de intervención tecnológica de MasAgro: proyecto piloto en el nodo de innovación (hub) trópico bajo de Chiapas", financiado por el CIMMYTMasAgro, México.

2 Universidad de Ciencias y Artes de Chiapas (UNICACH), Facultad de ingeniería, Sede Villacorzo. Carretera Villacorzo-Ejido Monterrey Km 3 CP. 30520, Chiapas, México. Tel.+965 6975325. manuel.hernandez@unicach.mx, luislarra2012@gmail.com

3 Universidad Autónoma de Chiapas (UNACH), Facultad de Ciencias Agronómicas. Carretera Ocozocoautla-Villaflores Km. 84.5, Apdo. postal 78, C.P.30470 Villaflores, Chiapas, México. Tel. +965 6521477.rosalesm@unach.mx; pinto_ruiz@yahoo.com.mx; Francisco.Guevara@ unach.mx (autor para correspondencia).

4 Instituto Nacional de Ciencias Agrícolas (INCA), Departamento de Genética y Mejoramiento Vegetal. Gaveta postal 1, C.P. 32700. San José de las Lajas, Mayabeque, Cuba. rodo2110@yahoo.com.mx 
de Valles Altos, caracterizada por la raza cónico Chalqueño y Elotes cónicos; el segundo se identificó por tener como ancestro común el Teocintle; el tercero contuvo el híbrido comercial H-MX3; y el cuarto comprendió los maíces locales de la REBISE y las variedades comerciales (Vs-536 y Vs-424).

Palabras clave: áreas protegidas, ISSR, polimorfismo, variabilidad genética. of reliability, however four major groups of varieties are clearly conformed. The first was named Valles Altos Corns, integrated by the conical Chalqueño and the conical Elotes races; the second is identified as having a common ancestor with Teocintle; the third is an improved hybrid by the H-MX3; and the fourth includes the REBISE local maize varieties and other commercial varieties (Vs-536 y Vs-424).

Keywords: protected areas, ISSR, polymorphism, genetic variability.

\section{INTRODUCCIÓN}

El maíz (Zea mays ssp. mays) es una especie que se caracteriza por su gran potencial de uso, gracias a su amplia diversidad genética. La variabilidad de sus alelos, es el reflejo de un largo proceso de domesticación en diferentes condiciones ambientales y culturales en Mesoamérica (Kato et al., 2009). Como resultado han surgido genotipos con características únicas, principalmente fenológicas, adaptativas y de uso alimenticio. Estos maíces son clasificados en diferentes razas. En México se conocen alrededor de 59 razas nativas, asociados a una larga tradición histórica de uso gastronómico, medicinal y religioso (Vázquez et al., 2003).

Botánicamente, el maíz es una planta alógama, monoica y con polinización típicamente influenciada por el viento (Ortiz et al., 2010). En la medida que las condiciones geográficas y de manejo favorecen los flujos de genes entre poblaciones contiguas, la estructura genética de una variedad no es estable (Louette, 1996). Por lo que, es común la existencia de superposiciones genéticas interraciales, que se ven reflejadas en sus características morfológicas, fenológicas y de potencial de uso de las variedades cultivadas (Perales y Hernández, 2005). En variedades nativas o tradicionales, estas características son controladas por la selección que ejercen los agricultores con base en una cultura y tradición de uso de cada material (Ortega et al., 2011).
En regiones donde se establecen nuevos grupos sociales, como sucede en algunas áreas naturales protegidas (ANP), las variedades cultivadas forman parte de nuevos procesos de construcción social. Un ejemplo lo constituye la Reserva de la Biosfera La Sepultura (REBISE), en Chiapas, México; que al igual que en otras partes del estado de Chiapas y de México, suceden varios fenómenos alrededor de los maíces. El germoplasma elegido depende de las necesidades de uso para el consumo local o comercial, debido a que existen diversos usos para el maíz, convergen en una zona tanto maíces tradicionales como mejorados (comerciales). Algunos de los maíces mejorados se conservan en forma de maíces segregados. Existe un intercambio permanente de semillas entre agricultores, dentro y/o entre comunidades (Louette, 1999). Como consecuencia de todos estos fenómenos ocurre una sustitución y combinación de los diferentes tipos de maíces (Bellon y Brush, 1994). Además, el proceso de selección de la semilla se realiza frecuentemente en el hogar y no en el campo (Keleman et al., 2009).

Lo anterior plantea que existe una constante modificación en la estructura genética de las poblaciones cultivadas, que hace posible una relación filogenética cercana entre germoplasmas; a su vez, dicha estructura influye en el potencial de uso y la formación de nuevas variedades locales. Esto demanda generar información sobre la cercanía filogenética y la relación con sus usos actuales y potenciales que aún es desconocida. 
Existe una serie de técnicas moleculares empleadas en la caracterización de los maíces y diferentes gramíneas, como los RFLP (restriction fragment length polymorphism), AFLP (amplified fragment length polymorphism), SSRs (simple sequence repeat), RAPD (random amplified polimorphic DNA), SNPs (single nucleotide polymorphisms) y los ISSR (Intersimple sequence repeat) o intermicrosatélites (Mbuya et al., 2012). Los ISSR son una técnica basada en $\mathrm{RCP}$, que ha demostrado ser eficiente por su alto grado de polimorfismo, además de ser rápida, sencilla y económica (Rentaría-Alcántara, 2007). En maíz, ha resultado ser eficaz para generar información acerca de la relación filogenética entre las variedades locales, híbridos y sus parientes silvestres (Luo et al., 2011).

El objetivo de este trabajo fue la caracterización molecular y filogenética de las poblaciones de maíces locales de la Reserva de la Biosfera La Sepultura (REBISE), México.

\section{MATERIALES Y MÉTODOS}

\section{Localización}

La investigación se realizó en el área natural protegida de la Reserva de la Biosfera La Sepultura (REBISE), en la porción oeste de la Sierra Madre de Chiapas (Figura 1). Se trabajó en nueve comunidades: El Triunfo, California, Tres Picos, Nueva Esperanza, Josefa Ortiz de Domínguez, La Sombra de la Selva, Ricardo Flores Magón y Villahermosa del municipio de Villaflores, y La Sierrita de Villacorzo, en la región VI Frailesca del Estado de Chiapas, durante el ciclo agrícola 2012-2013 (Cuadro 1).

Se seleccionó la región Frailesca por su importante contribución a la producción de maíz en el Estado de Chiapas, y a la REBISE porque en ella los productores aún manejan y conservan materiales genéticos locales (PROMAC, 2011). Las comunidades se eligieron por ser los principales nichos agroecológicos donde se

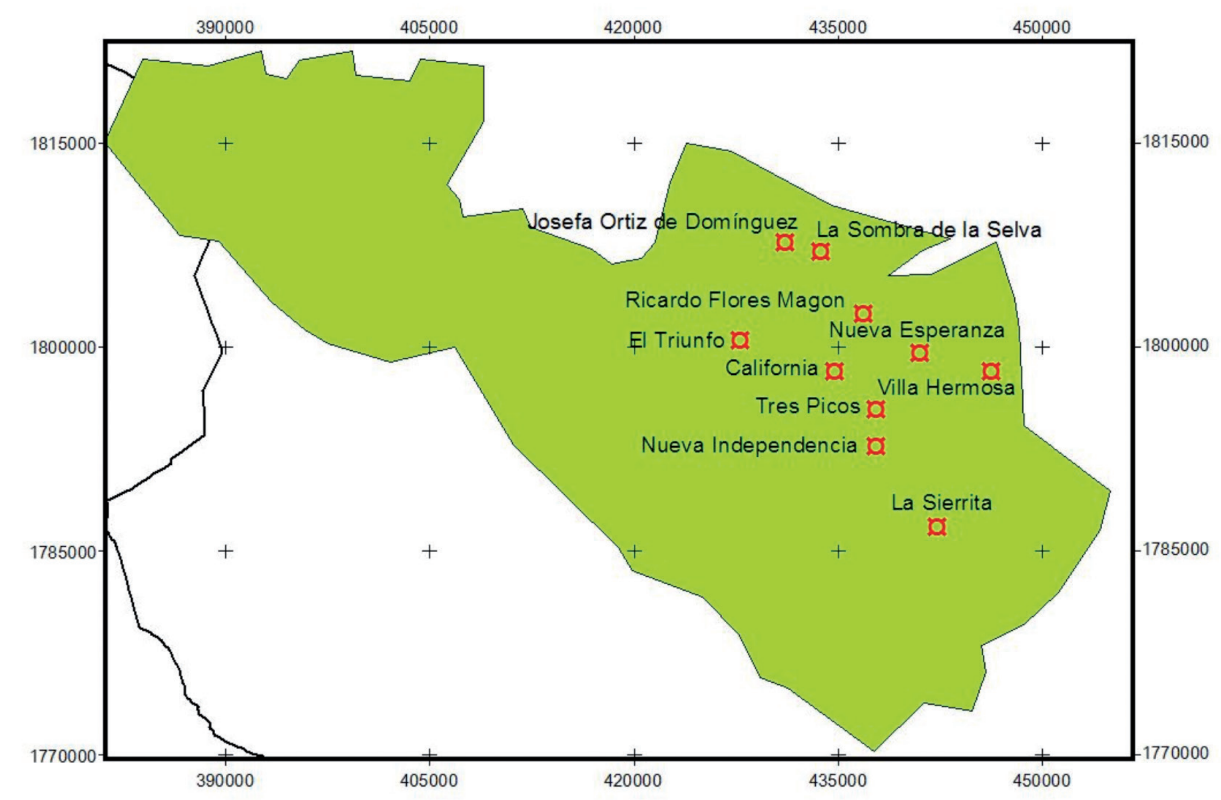

Figura 1. Área natural protegida de la Reserva de la Biosfera La Sepultura (REBISE), en la porción Oeste de la Sierra Madre de Chiapas, México, donde se obtuvieron los maíces locales empleados en la caracterización molecular. 2013.

Figure 1. Natural protected Natural protected area: Reserva de la Biosfera La Sepultura (REBISE) in the Western part of the Sierra Madre of Chiapas, Mexico, where in 2013 the local maize samples were collected for the molecular characterization. 
Cuadro 1. Ubicación geográfica de las nueve comunidades muestreadas, en el área natural protegida de la Reserva de la Biosfera La Sepultura (REBISE), en la porción oeste de la Sierra Madre de Chiapas, México, donde se obtuvieron los maíces locales empleados en la caracterización molecular en 2013.

Table 1. Geographical localization of the nine studied communities within the natural protected area: Reserva de la Biosfera La Sepultura (REBISE) in the Western part of the Sierra Madre of Chiapas, Mexico; where the local maize samples were collected for the molecular characterization in 2013.

\begin{tabular}{lccc}
\hline \multirow{2}{*}{ Comunidad } & \multicolumn{2}{c}{ Posición } & Altitud ${ }^{\mathbf{1}}(\mathbf{m})$ \\
\cline { 2 - 4 } & Latitud norte & Longitud oeste & \\
\hline El Triunfo & $16^{\circ} 16^{\prime} 49^{\prime \prime}$ & $93^{\circ} 40^{\prime} 38^{\prime \prime}$ & 1040 \\
California & $16^{\circ} 15^{\prime} 36^{\prime \prime}$ & $93^{\circ} 36^{\prime} 44^{\prime \prime}$ & 960 \\
Tres Picos & $16^{\circ} 14^{\prime} 05^{\prime \prime}$ & $93^{\circ} 35^{\prime} 00^{\prime \prime}$ & 998 \\
Nueva Esperanza & $16^{\circ} 16^{\prime} 20^{\prime \prime}$ & $93^{\circ} 33^{\prime} 14^{\prime \prime}$ & 802 \\
Josefa Ortiz de Domínguez & $16^{\circ} 20^{\prime} 44^{\prime \prime}$ & $93^{\circ} 38^{\prime} 49^{\prime \prime}$ & 920 \\
La Sombra de la Selva & $16^{\circ} 20^{\prime} 23^{\prime \prime}$ & $93^{\circ} 37^{\prime} 19^{\prime \prime}$ & 839 \\
Ricardo Flores Magón & $16^{\circ} 17^{\prime} 55^{\prime \prime}$ & $93^{\circ} 35^{\prime} 33^{\prime \prime}$ & 719 \\
Villahermosa & $16^{\circ} 15^{\prime} 36^{\prime \prime}$ & $93^{\circ} 30^{\prime} 17^{\prime \prime}$ & 1042 \\
La Sierrita & $16^{\circ} 09^{\prime} 26^{\prime \prime}$ & $93^{\circ} 32^{\prime} 30^{\prime \prime}$ & 914 \\
\hline
\end{tabular}

${ }^{1}$ Tiene como referencia el centro del área urbana. Fuente: INEGI (2010)./ The center of the urban area is taken as the main point of reference. Source: INEGI (2010).

cultivan maíces locales (Perales y Hernández, 2005; Aguilar, 2010; PROMAC, 2011).

\section{Características generales del área de estudio}

La topografía de la REBISE presenta diferentes altitudes, lo que ocasiona variabilidad en las condiciones climáticas, las características de los suelos, la accesibilidad de corrientes de agua y la adaptación de diferentes prácticas de manejo en la agricultura. Las características ambientales cambian desde la zona de mayor altitud (el cerro Tres Picos) hasta las áreas planas, en dirección a la costa del Pacífico y hacia la Depresión Central (Velasco et al., 2005).

En general, las condiciones climáticas predominantes son: 1) $\mathrm{A}(\mathrm{C}) \mathrm{m}(\mathrm{w})$ o semicálido húmedo. Este clima se caracteriza por presentar temperaturas medias anuales de $18{ }^{\circ} \mathrm{C}$ y abundantes lluvias en verano; se encuentra presente en la mayoría de las comunidades, con excepción de Nueva Independencia. 2) AW2(w) o cálido subhúmedo; presenta temperaturas medias anuales mayores de $22{ }^{\circ} \mathrm{C}$, lluvias en verano y sequías en invierno. Es característico de los ejidos: California, Tres Picos, Nueva Esperanza, Ricardo Flores Magón, La Sombra de la Selva, Josefa Ortiz de Domínguez y La Sierrita. 3) C(m)(w) o templado húmedo. Presenta temperaturas medias anuales de 12$18{ }^{\circ} \mathrm{C}$ y abundantes lluvias en verano; únicamente se encuentra en el ejido Nueva Independencia.

\section{Caracterización molecular}

\section{Material genético}

Se utilizaron diecinueve materiales locales de la REBISE, colectados durante el ciclo agrícola 20122013 (Cuadro 2 y Cuadro 3). La colecta se realizó en la parcela de cada agricultor. El manejo respondió a la forma en que lo hace cada agricultor. Los materiales fueron seleccionados por su alta aceptación entre los agricultores; presentan mayor distribución y han permanecido por más tiempo en la región 
Cuadro 2. Procedencia de las semillas de los diecinueve maíces locales colectados en el año 2013 en las comunidades de la Reserva de la Biosfera La Sepultura (REBISE), Chiapas, México.

Table 2. Origin of the nineteen local maize seed samples collected during 2013 at the communities located in the Reserva de la Biosfera La Sepultura (REBISE), Chiapas, Mexico.

\begin{tabular}{llcc}
\hline Comunidad & Agricultor donante & Maíz cultivado $^{1}$ & Acrónimo \\
\hline California & Mario Hernández López & Amarillo & AmarilloC \\
Ricardo Flores Magón & Rafael Cruz Ruíz & Amarillo & AmarilloRFM \\
Villahermosa & Javier de Jesús López Castro & Amarillo & AmarilloV \\
Josefa Ortiz de Domínguez & Arturo de la Cruz Sánchez & Amarillo & AmarilloJOD \\
California & Cornelio Santos Avendaño & Precoz & PrecozC \\
Tres Picos & Silverio Moguel Cruz & Precoz & PrecozTP \\
Tres Picos & Neftalí Cruz Santos & Jarocho & JarochoTP \\
El Triunfo & Omar Torres & Jarocho & JarochoET \\
Ricardo Flores Magón & Rafael Cruz Ruíz & Jarocho & JarochoRFM \\
Villahermosa & Ponciano Castro Ozuna & Jarocho & JarochoV \\
Ricardo Flores Magón & Segundo Gómez López & Chimbo & Chimbito \\
Ricardo Flores Magón & Segundo Gómez López & Pollito & Pollito \\
Nueva Esperanza & Francisco Ramón Sixto Morales & Olote-Rojo & ORojo \\
Tres Picos & Fernando Díaz López & Huesito & Huesito \\
La Sombra de la Selva & Onésimo Hernández Gutiérrez & Morales & Morales \\
California & Mariano Ruíz Ruíz & Maíz Negro & MNegro \\
California & Juan Carlos Cruz Pérez & Crema & Crema \\
La Sierrita & Migue Ángel Clemente Ramírez & Rafael Cruz Ruíz & SGregorio \\
Ricardo Flores Magón & & Tornamil & \\
\hline
\end{tabular}

${ }^{1}$ Es identificado por su nombre común o nombre como se conoce por los propios agricultores. Muchas veces son nombres autodescriptivos o que se relacionan con su origen (por ej. El nombre o apellido de la persona o agricultor que se considera fue el responsable de difundirlo) / It is identified by the common name or the way it is named by the farmers. Many times they have descriptive names or closely related to their origin (i.e. the name or last name of the person or farmer who was responsible for its distribution).

(Hernández-Ramos, 20014). Además, se incluyeron, dos variedades mejoradas comerciales (Vs-536 y Vs-424), un híbrido comercial (H-MX3), un material recientemente introducido a la REBISE (Costeño), dos materiales de Tlaxcala, México (de las razas cónico chalqueño y elotes cónicos) y un teocintle (Zea mays ssp. Mexicana), del estado de Tlaxcala, México. Estos materiales adicionales fueron utilizados como testigos para comparar las distancias genéticas. Los maíces de Tlaxcala y teocintle representan, por su lejanía geográfica y morfológica, un ideal testigo para comparar. Para su identificación se tomaron los criterios establecidos por Wellhausen et al. (1951). En el caso del teocintle la indentificación se hizo por comparación del material colectado con información presente en el CIMMYT. En total se utilizaron veintiséis materiales diferentes. El análisis molecular se realizó durante el año 2013 en el Laboratorio de Recursos Fitogenéticos en la Facultad de Ciencias Agronómicas Campus V de la Universidad Autónoma de Chiapas (UNACH), Villaflores, Chiapas, México.

\section{Extracción del ADN}

La extracción del ADN se basó en la metodología de Asemota (1995; modificado por Solís, 2012). Para conocer la cantidad exacta de muestra a usar, previo al estudio, se realizó una prueba de extracción de ADN. Se utilizaron tres pesos diferentes de 50, 100 y 150 mg de hoja de maíz y como control positivo, 80 mg de 
Cuadro 3. Descripción general de los diecinueve maíces locales colectados en el año 2013 en las comunidades de la Reserva de la Biosfera La Sepultura (REBISE), Chiapas, México.

Table 3. General description of the nineteen local maize seed samples collected during 2013 at the communities located in the Reserva de la Biosfera La Sepultura (REBISE), Chiapas, Mexico.

\begin{tabular}{|c|c|c|c|}
\hline \multirow{2}{*}{$\begin{array}{l}\text { Maíz local } \\
\text { (acrónimo) }\end{array}$} & \multicolumn{2}{|c|}{ Razas relacionadas $^{1,2}$} & \multirow[t]{2}{*}{ Características de uso $^{1}$} \\
\hline & Primaria & Secundarias & \\
\hline AmarilloC & Tepecintle & Tuxpeño, olotillo & \multirow{4}{*}{$\begin{array}{l}\text { Se destinan al autoconsumo. En el caso de los que tienen su } \\
\text { origen en el tuxpeño también al mercado. Son resistentes } \\
\text { a la pudrición de mazorca. Se emplean en la alimentación } \\
\text { animal y se consideran buenos para producción de forraje } \\
\text { y rastrojo. }\end{array}$} \\
\hline AmarilloRFM & Tuxpeño & - & \\
\hline AmarilloV & Tuxpeño & Olotillo, tepecintle & \\
\hline AmarilloJOD & Olotillo & Tepecintle & \\
\hline PrecozC & Tuxpeño & Vandeño & \multirow{2}{*}{$\begin{array}{l}\text { Son considerados resisten a la pudrición de mazorca y al } \\
\text { viento. Se emplean para el autoconsumo y venta local. Muy } \\
\text { buenos en la producción de alimentos. Se utilizan para la } \\
\text { alimentación animal, pero no son muy buenos para rastrojo. }\end{array}$} \\
\hline PrecozTP & Tuxpeño & - & \\
\hline JarochoTP & Tuxpeño & Vandeño & \multirow{4}{*}{$\begin{array}{l}\text { Requieren de una mejor fertilización. Se emplea para } \\
\text { el autoconsumo y la venta local. Son ideales para la } \\
\text { producción de elotes y forraje verde. Se utilizan en la } \\
\text { alimentación de animales. }\end{array}$} \\
\hline JarochoET & Tuxpeño & Vandeño & \\
\hline JarochoRFM & Tuxpeño & - & \\
\hline JarochoV & Olotillo & Tuxpeño, vandeño, tepecintle & \\
\hline Chimbito & - & - & $\begin{array}{l}\text { Es un material que se considera resistente al viento. Se } \\
\text { emplea únicamente para autoconsumo. Se utiliza para la } \\
\text { alimentación animal. Es bueno para rastrojo. }\end{array}$ \\
\hline Pollito & - & - & $\begin{array}{l}\text { Presenta poca resistencia a la pudrición de mazorca, } \\
\text { pero resistente al viento. Se emplea únicamente para } \\
\text { autoconsumo. }\end{array}$ \\
\hline ORojo & Tuxpeño & - & $\begin{array}{l}\text { Es un material que resiste muy bien al picado durante el } \\
\text { almacenamiento; pero requiere de una mayor fertilización. }\end{array}$ \\
\hline Huesito & Olotillo & Tuxpeño & $\begin{array}{l}\text { Requiere una mejor fertilización. Es un material muy duro } \\
\text { al momento de desgranar. Es empleado para autoconsumo. }\end{array}$ \\
\hline Morales & Tuxpeño & - & $\begin{array}{l}\text { Se emplea para autoconsumo y mercado. También en la } \\
\text { alimentación animal. Es bueno para resistencia al viento y } \\
\text { pudricion de mazorca. }\end{array}$ \\
\hline MNegro & Olotillo & Tuxpeño, vandeño & $\begin{array}{l}\text { Se considera bueno para autoconsumo. No se emplea para } \\
\text { la alimentación animal. }\end{array}$ \\
\hline Crema & Tuxpeño & - & $\begin{array}{l}\text { Requiere una mejor fertilización. Se emplea únicamente } \\
\text { para autoconsumo. Es apremiado para la alimentación } \\
\text { familiar y de animales. }\end{array}$ \\
\hline SGregorio & Tuxpeño & - & $\begin{array}{l}\text { Se emplea para autoconsumo y mercado. También en la } \\
\text { alimentación animal. }\end{array}$ \\
\hline Tornamil & Tuxpeño & - & $\begin{array}{l}\text { Se emplea para autoconsumo y mercado. También en la } \\
\text { alimentación animal. }\end{array}$ \\
\hline
\end{tabular}

${ }^{1}$ La presente información fue parte de los resultados de la caracterización socio-agronómica realizada en la tesis de posgrado del primero autor de este trabajo titulada "Maíces locales con potencial de uso múltiple en un área natural protegida de Chiapas" / This information was obtained as part of the results from a socio-agronomic characterization made for the MSc thesis of the first author of this paper and was entitled: local maize with multiple uses in a natural protected area of Chiapas.

${ }^{2}$ Para la identificación de los materiales se tomaron los criterios establecidos por Wellhausen et al. (1951). Descritos para las razas de maíz en México / For the identification of collected materials, the criteria defined by por Wellhausen et al. (1951) were used. They are specifically described for the Mexican maize races. 
Jatropha curcas L. Los pesos 100 y $150 \mathrm{mg}$ presentaron ADN de mejor calidad. Por lo cual, para la extracción del ADN de las muestras de interés se utilizó de 115 $\pm 15 \mathrm{mg}$ de muestra fresca (hoja libre de impurezas y daños) por plántula. Se realizaron dos repeticiones por germoplasma. Para los materiales con poco ADN se repitió la extracción.

\section{Cebadores utilizados}

Se realizó una prueba con veintiún cebadores ISSR; de los cuales, para el análisis de caracterización, se utilizó únicamente los diez que amplificaron y presentaron polimorfismo en los maíces estudiados. En el Cuadro 4 se describen los diez cebadores utilizados.

\section{Amplificación del ADN}

En el Cuadro 5 se presenta la mezcla de reacción para la amplificación del ADN. Este procedimiento se realizó con la técnica de reacción en cadena de polimerasa (RCP), con un volumen de reacción de $25 \mu 1$ de solución total para cada muestra. Las reacciones se realizaron por duplicado, para cada una de las muestras.

\section{Electroforesis}

Se emplearon geles de agarosa al 1,5\% en solución TAE 1X. En la extracción del ADN genómico, para fines de corroborar su obtención y calidad, se utilizó al $1 \%$. En el gel se cargaron los $25 \mu 1$ de la mezcla de reacción. Se utilizaron dos marcadores de peso molecular: 1) Marcador de 100 pb de ADN (diez bandas de $100 \mathrm{pb}$ hasta $1000 \mathrm{pb}$ ) y 2) Marcador de 1 $\mathrm{Kb}$ de ADN (catorce bandas de 200 pb hasta 10000 $\mathrm{pb).} \mathrm{Para} \mathrm{visualizar} \mathrm{las} \mathrm{veintiséis} \mathrm{muestras} \mathrm{duplicadas,}$ se requirió dos geles por cada cebador utilizado. La electroforesis se realizó en una cámara OWL modelo A2 a $80 \mathrm{~V}$, durante 150 minutos. Para la tinción de geles se utilizó solución de GelRed 1X; después del corrimiento electroforético los geles se incubaron durante $40 \mathrm{~min}$. Posteriormente, se fotodocumentaron en un transiluminador UV del tipo Gel Doc.

Cuadro 4. Secuencia genética y temperatura de hibridación de los diez cebadores ISSR empleados durante 2013 en la caracterización molecular de diecinueve materiales locales de maíz de la Reserva de la Biosfera La Sepultura (REBISE), Chiapas, México.

Table 4. Gene sequence and hybridization temperature of the ten ISSR primers used during 2013 for the molecular characterization of nineteen local maize materials from the Reserva de la Biosfera La Sepultura (REBISE), Chiapas, Mexico.

\begin{tabular}{llc}
\hline Cebador & \multicolumn{1}{c}{ Secuencia* $^{*}$} & $\begin{array}{c}\text { Temperatura de } \\
\text { hibridación }\end{array}$ \\
\hline$\left.{ }^{\circ} \mathbf{C}\right)$
\end{tabular}

* G: guanina; A: adenina; C: citosina; Y: citosina o timina / G: guanine; A: adenine; C: cytosine; Y: cytosine or thymine. 
Cuadro 5. Mezcla de reacción para la amplificación del ADN empleado durante 2013 en la caracterización molecular de diecinueve maíces locales de la Reserva de la Biosfera La Sepultura (REBISE), Chiapas, México.

Table 5. Reaction mixture for amplifying DNA employed during 2013 in the molecular characterization of nineteen local maize materials from the Reserva de la Biosfera La Sepultura (REBISE), Chiapas, Mexico.

\begin{tabular}{lc}
\hline Reactivo & Cantidad $(\boldsymbol{\mu l})$ \\
\hline ADN molde & 1,0 \\
Solución amortiguadora $\left(\mathrm{NH}_{4} 10 \mathrm{X}\right)$ & 2,5 \\
$\mathrm{MgCl}_{2}(50 \mathrm{mM})$ & 1,5 \\
$\mathrm{dNTP}$ s $(1 \mathrm{mM})$ & 5,0 \\
Cebador $(1 \mu \mathrm{g} / \mu \mathrm{l})$ & 0,4 \\
$\mathrm{ADN}$ polimerasa $(5 \mathrm{U} / \mu \mathrm{l})$ & 0,1 \\
$\mathrm{H}_{2} \mathrm{O}$ & 14,5 \\
Total & 25,0 \\
\hline
\end{tabular}

\section{Análisis estadísticos}

Se realizó un análisis de similitud mediante coeficiente de asociación DICE (Dice, 1945), y el coeficiente de similitud simple (SM, por sus siglas en inglés) (Sokal y Michener, 1958). Se hizo un análisis de agrupamiento, método aritmético de grupo de pares no ponderados (UPGMA, por sus siglas en inglés) (Sneath y Sokal, 1973) y análisis de coordenadas principales. Para la realización de los análisis se empleó el programa NTSYSpc 2.2.

\section{RESULTADOS Y DISCUSIÓN}

\section{Calidad del AND genómico}

Con la metodología empleada se obtuvo ADN genómico de buena calidad y cantidad suficiente para la amplificación de cebadores tipo ISSR (Figura 2). La
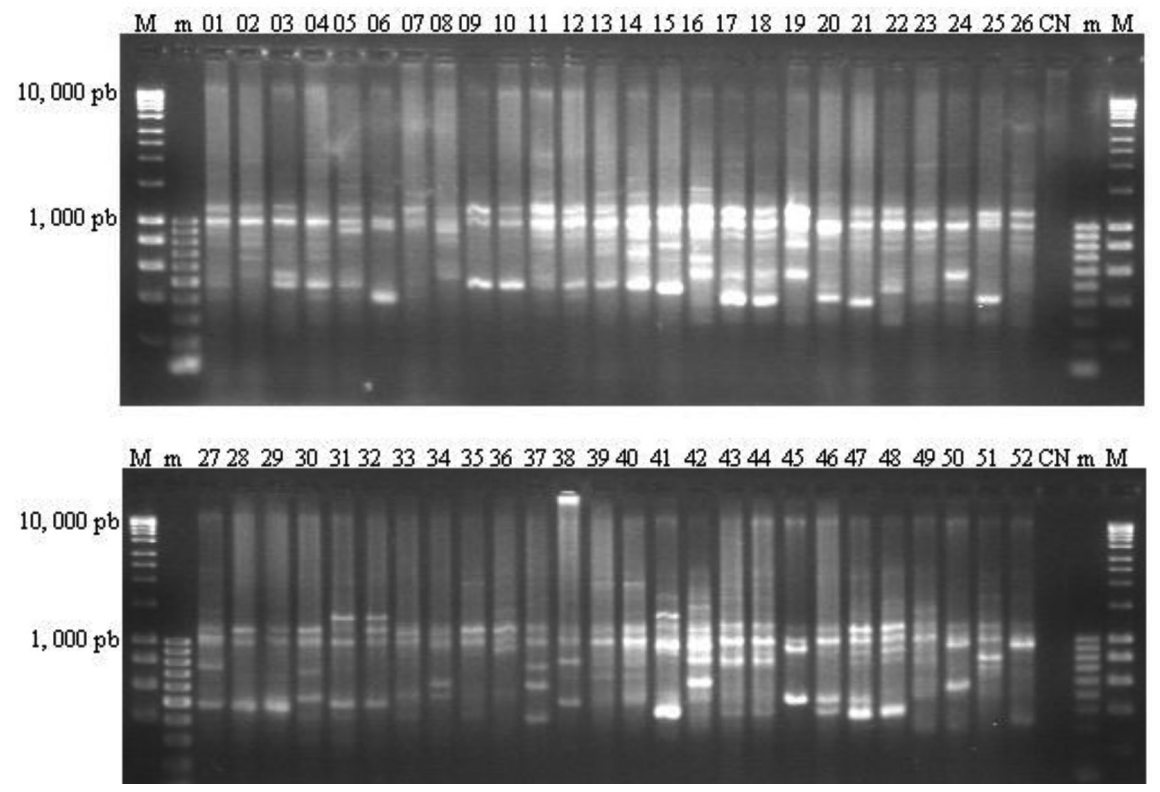

Figura 2. Amplificación del UBC868 en diecinueve maíces locales de la Reserva de la Biosfera La Sepultura (REBISE), Chiapas, México. 2013.

Figure 2. Amplification of UBC868 in nineteen local maize materials from the Reserva de la Biosfera La Sepultura (REBISE), Chiapas, Mexico. 2013. 
numeración del 01 a 40 representó (por duplicado) a cada maíz local de la REBISE.

\section{Amplificación y polimorfismo de cebadores ISSR}

El éxito de la amplificación de los cebadores depende de las condiciones de RCP y del tipo de secuencia de cada cebador (Pradeep et al., 2002). En este análisis, de los diez cebadores utilizados, ocho se formaron por los dinucleótidos $(\mathrm{AG})_{8},(\mathrm{AC})_{8}$ y $(\mathrm{GA})_{8}$, uno por el trinucleótido (GAA) ${ }_{6}$ y otro por el tetranucleótido (GACA) $)_{4}$. En el caso de los dinucleótidos, siete presentaron anclaje $(\mathrm{C}, \mathrm{YT}$, YC y YG) en el extremo 3'. Los dinucleótidos y el trinucleótido fueron más polimórficos que el tetranucleótido.En los dinucleótidos, las secuencias AG y AC tuvieron mayor número de bandas amplificadas que la secuencia GA; esta mayor frecuencia de AG y AC ocurrió en el trinucleótido GAA. En concordancia, se ha reportado que en maíz, regiones de GA (Blair et al., 1999; Carvalho et al. 2002; Do-Amaral-Júnior et al., 2011) y AG son más comunes, esta última como la más frecuente (Taramino y Tingey, 1996). En otras gramíneas, por ejemplo, en arroz el AG y GA amplifican mejor (Joshi et al., 2000), y en trigo amplifica el AC (McGregor et al., 2000).

En los cebadores utilizados, el I9 amplificó catorce bandas (100\% polimórficas). En el UBC857 y UBC827 el número de fragmentos amplificados fue de once y doce, de los cuales el 81,8 y $83,3 \%$ fueron polimórficas, respectivamente. El UBC834 amplificó el mayor número de bandas (16), con 93,7\% de polimorfismo; y el I4 amplificó diez bandas con $70 \%$ de polimorfismo. Para el UBC841 se encontró $87,5 \%$ de polimorfismo de ocho bandas amplificadas. Lo anterior concuerda con un estudio hecho por DoAmaral-Júnior et al. (2011), donde cebadores con secuencias $(\mathrm{AG})_{8} \mathrm{YT},(\mathrm{GA})_{8} \mathrm{YC}$ y $(\mathrm{CA})_{8} \mathrm{YG}$ mostraron $80,91,7$ y $100 \%$ de polimorfismo de dieciséis, doce y nueve bandas amplificadas, respectivamente.

El trinucleótido (GAA) 6 amplificó dieciséis bandas, todas polimórficas. Berilli et al. (2011) reportaron para esta secuencia $72,73 \%$ de polimorfismo de once bandas amplificadas en maíz. En contraste, en arroz no presentó amplificación (Joshi et al., 2000). El tetranucleótido (GACA) presentó una banda polimórfica de cuatro amplificadas. En maíz, Carvalho et al. (2002) reportaron resultados similares en amplificación (cinco bandas amplificadas), pero diferente en polimorfismo (80\%). En otras gramíneas como el arroz, no presentó amplificación (Blair et al., 1999), y en Pennisetum glaucum se reportaron once bandas amplificadas, $72,73 \%$ polimórficas (Kumar et al., 2006).

En total se obtuvieron 113 fragmentos, cuyos pesos moleculares fluctuaron entre 150 y $2200 \mathrm{pb}$. De los cuales 97 fragmentos $(85,8 \%)$ fueron polimórficos. Los cebadores UBC834, I9 y UBC868 conformados por las secuencias $(\mathrm{AG})_{8} \mathrm{YT},(\mathrm{AC})_{8}$ y $(\mathrm{GAA})_{6}$, respectivamente, fueron los que mejor amplificaron (Cuadro 6). El resto de los dinucleótidos permitieron un alto grado de diferenciación de los materiales analizados. El tetranucleótido mostró ser un marcador poco común en maíz y específico para algunas gramíneas. En este sentido, se ha reportado que el número de fragmentos amplificados y polimórficos obedecen al tipo y posición del anclaje (Pradeep et al., 2002). También se conoce que el potencial de las secuencias para generar información genética a través de los fragmentos polimórficos, depende de la frecuencia con que se encuentra y distribuyen en el genoma de la especie (Morgante y Olivieri, 1993).

En otras especies, algunos cebadores que se utilizaron en este estudio se han asociado a genes específicos. En el arroz el $(\mathrm{AG})_{8} \mathrm{YC}$ se asocia a

Cuadro 6. Polimorfismo de los cebadores analizados durante 2013 en la caracterización molecular de diecinueve maíces locales de la Reserva de la Biosfera La Sepultura (REBISE), Chiapas, México.

Table 6. Polymorphism of primers analyzed during 2013 for the molecular characterization of nineteen local maize materials from the Reserva de la Biosfera La Sepultura (REBISE), Chiapas, Mexico.

\begin{tabular}{lcccc}
\hline Cebador & Secuencia & $\begin{array}{c}\text { Bandas po- } \\
\text { limórficas }\end{array}$ & $\begin{array}{c}\text { Bandas mo- } \\
\text { nomórficas }\end{array}$ & $\begin{array}{c}\text { Total de } \\
\text { bandas }\end{array}$ \\
\hline UBC811 & $(\mathrm{GA})_{8} \mathrm{C}$ & 8 & 2 & 10 \\
UBC827 & $(\mathrm{AC})_{8} \mathrm{G}$ & 10 & 2 & 12 \\
UBC834 & $(\mathrm{AG})_{8} \mathrm{YT}$ & 15 & 1 & 16 \\
UBC835 & $(\mathrm{AG})_{8} \mathrm{YC}$ & 10 & 2 & 12 \\
UBC841 & $(\mathrm{GA})_{8} \mathrm{YC}$ & 7 & 1 & 8 \\
UBC857 & $(\mathrm{AC})_{8} \mathrm{YG}$ & 9 & 2 & 11 \\
UBC868 & $(\mathrm{GAA})_{6}$ & 16 & 0 & 16 \\
UBC873 & $(\mathrm{GACA})_{4}$ & 1 & 3 & 4 \\
I4 & $(\mathrm{AG})_{8} \mathrm{C}$ & 7 & 3 & 10 \\
I9 & $(\mathrm{AC})_{8}$ & 14 & 0 & 14 \\
Total & - & $\mathbf{9 7}$ & $\mathbf{1 6}$ & $\mathbf{1 1 3}$ \\
\hline
\end{tabular}


la producción de semilla (Akagi et al., 1996). En garbanzo se han encontrado asociaciones al gen de resistencia a Fusarium, de los cuales (GAA) 6 se asoció al gen de resistencia de las razas 4 y 5 de Fusarium; y la secuencia (AC) ${ }_{8} \mathrm{YG}$, para la raza 4 de Fusarium (Ratnaparkne et al., 1998). En maíz, los cebadores $(\mathrm{GA})_{8} \mathrm{YC},(\mathrm{AG})_{8} \mathrm{YT},(\mathrm{AC})_{8} \mathrm{YG}$ y $(\mathrm{GACA})_{4}$ se emplearon exitosamente, junto a otros, en la diferenciación de materiales por sus características morfológicas y químicas, principalmente. Por ejemplo, en Brasil se caracterizaron 81 variedades de maíces y se encontró que el agrupamiento obtenido se relacionó con el color de grano y el tiempo de floración (Carvalho et al., 2002). Mbuya et al. (2012) seleccionaron genotipos de alta calidad proteica (QPM), y Do-Amaral-Júnior et al. (2011) evaluaron la variabilidad genética de maíces palomeros. La existencia de relaciones de estos cebadores con características morfológicas y químicas de los maíces, revela su aptitud para caracterizar, seleccionar y diferenciar variedades de maíces y sus parientes, e inferir en expresiones de adaptación, resistencia y potencial de uso de cada material analizado. Sin embargo, son necesarios más estudios posteriores para encontrar mayores indicadores genéticos y relaciones más específicas entre cebadores y las características intrínsecas y extrínsecas de los maíces.

El alto polimorfismo encontrado en los maíces locales de la REBISE, mostró que existe variabilidad genética. Las bandas monomórficas indicaron sus similitudes como especie, o probablemente características específicas de las plantas. La similitud entre los materiales evaluados por la presencia de bandas polimórficas entre poblaciones, pueden deberse a su igualdad como variedades, razas, especie o al efecto de la combinación genética, resultado de su polinización cruzada. Las diferencias pueden estar ligadas a aspectos particulares de las variedades, razas o efecto del medio ambiente (adaptabilidad) donde se desarrollan. Para el análisis de similitud y agrupamiento, se utilizaron todas las bandas encontradas, tanto polimórficas como monomórficas.

\section{Similitud de los maíces locales}

El coeficiente de asociación DICE (Dice, 1945), considera que la presencia de un valor ofrece mayor información que su ausencia. La ausencia puede deberse a diferentes factores y no necesariamente refleja diferencias genéticas entre muestras. El coeficiente de igualdad simple (SM, por sus siglas en inglés) (Sokal y Michener, 1958) asume que no existe diferencia cuando ambas muestras presentan el mismo valor (Legendre y Legendre, 1983). Ambos coeficientes fueron eficientes en diferenciar los maíces locales de la REBISE de los testigos, principalmente del teocintle, de los maíces de las razas elotes cónicos y cónico chalqueño, y del híbrido H-MX3. Sin embargo, aunque el coeficiente DICE propició mayor lejanía entre los materiales, el coeficiente SM proporcionó distancias genéticas que permitieron una mejor agrupación de los germoplasmas y sus repeticiones.

En general, los germoplasmas analizados mostraron un coeficiente SM de 0,611 a 0,938 . Entre repeticiones de cada germoplasma este coeficiente fluctuó de 0,814 a 0,938 , y entre germoplasmas de 0,681 a 0,912 . Los coeficientes más bajos $(0,611$ a 0,805$)$ los presentaron los testigos teocintle, elotes cónicos, cónico chalqueño y H-MX3. Este comportamiento parece ser explicado por el origen racial de cada muestra utilizada, lo que ha permitido una diferenciación muy clara entre testigos y variedades locales. Por ejemplo, se pudieron diferenciar claramente de los maíces locales de la REBISE: 1) el teocintle, que es una especie silvestre y pariente del maíz; 2) las razas elotes cónicos y cónico chalqueño, que son maíces de la región de Tlaxcala, México; y 3) el H-MX3, que es un maíz híbrido con un largo proceso de mejoramiento genético para regiones tropicales.

Las variedades sintéticas mejoradas (Vs-536 y Vs-424) presentaron mayores similitudes con los maíces locales de la REBISE $(0,885)$. En este caso, pueden existir dos explicaciones para la presencia de un mismo alelo en un locus dado en germoplasmas diferentes: 1) que compartan un ancestro común, por ejemplo, estén relacionados con una misma raza y/o; 2) que lo hayan adquirido por causas externas, como es el flujo de genes (Morales, 2002).

En el caso particular del teocintle, el amplio rango de similitud $(0,611$ a 0,805$)$ no implica una menor o mayor relación con cualquier variedad de maíz evaluada (locales y testigos); es decir, las similitudes entre estas subespecies pueden estar relacionadas con la capacidad de adaptarse a ciertos ambientes, y no necesariamente a la existencia de introgresiones genéticas (Hufford et al., 2013).

Un índice de similitud bajo no implica que los individuos pertenezcan a diferentes taxones (Pérez et al., 2010). Por lo cual, las diferencias entre las 
variedades locales de la REBISE puede deberse a los diversos procesos de adaptación y selección que han sufrido como variedades, y no necesariamente que sean diferentes razas. Es decir, son procesos que han permitido conservar genes específicos que propician características de importancia para los agricultores, como resistencias a diferentes factores ambientales y usos locales donde son cultivados.

La diversidad genética de maíces locales puede ser el reflejo del resultado de la interacción de factores de manejo, distancia geográfica, selección constante y flujo de genes. Sin embargo, es necesario realizar estudios más específicos que ayuden a comprender mejor los efectos antropogénicos en los cambios genéticos de los germoplasmas locales.

\section{Agrupamiento de los maíces locales}

Los marcadores ISSR permitieron conocer la lejanía entre los materiales. El dendograma obtenido (Figura 3) mostró una correlación cofenética relativamente baja $(r=0,721)$, lo cual se traduce en que las distancias representadas en el dendograma tienen un grado de confiabilidad bajo. Según Gutiérrez et al. (2009) y Durán-Hernández et al. (2011) los valores aceptables para un coeficiente de correlación cofenética, desde el punto de vista de la reproducibilidad, debe ser $\geq 0,8$; sin embargo, aun así se logró agrupar en forma clara los materiales analizados.

Los resultados mostraron que los materiales evaluados se integraron en cuatro grupos principales. El primer grupo se integró por los testigos de la raza

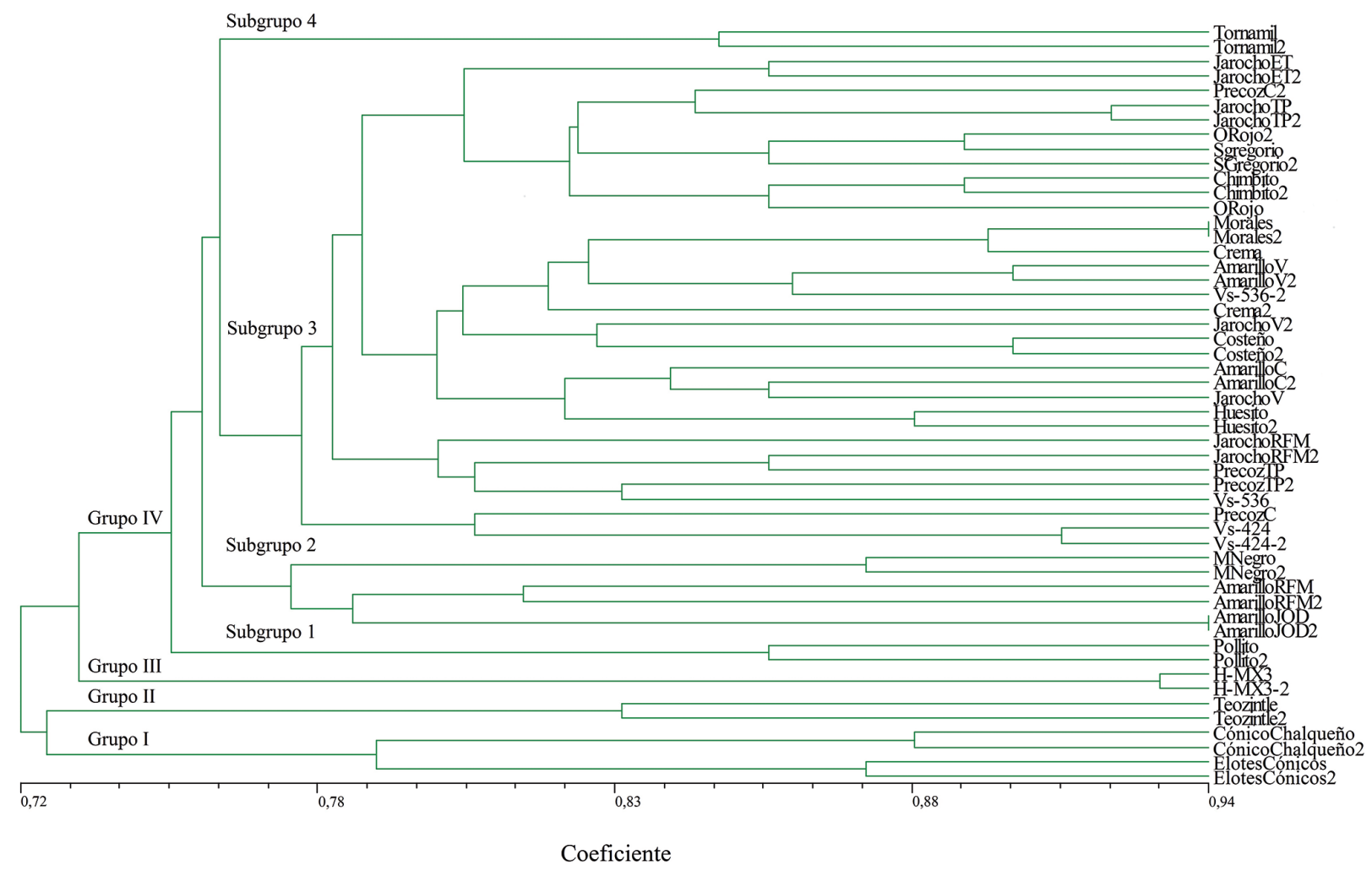

Figura 3. Dendograma obtenida con la matriz de similitud que evidencia la distancia genética entre las muestras analizadas durante 2013 de diecinueve maíces locales de la Reserva de la Biosfera La Sepultura (REBISE), Chiapas, México.

Figure 3. Dendrogram obtained with the similarity matrix that shows the genetic distance between the nineteen analyzed samples during 2013 of local maize from the Reserva de la Biosfera La Sepultura (REBISE), Chiapas, Mexico. 
cónico chalqueño y elotes cónicos (1); el segundo grupo por el teocintle (2); el tercer grupo por el H-MX3 (3); y el cuarto grupo comprendió los maíces locales de la REBISE y las variedades mejoradas (4). El grupo cuatro se dividió en cuatro subgrupos; y el tercer subgrupo en otros cuatro subsubgrupos.

En el análisis de los grupos principales se estableció un límite inter-racial $(0,75)$ creado por los testigos teocintle, elotes cónicos, cónico chalqueño y H-MX3. En el grupo cuatro los subgrupos formados se explicaron por posibles interacciones de las principales razas reportadas para la región Frailesca, Chiapas; como son tuxpeño, olotillo, vandeño y tepecintle, entre otras (Perales y Hernández, 2005; PROMAC, 2011). Una característica particular de este cuarto grupo fue que las razas mencionadas para la frailesca también presentaron una estrecha relación interracial (González et al., 2013); posiblemente relacionado con que todas estas razas provengan de orígenes similares, y que algunos sean resultados de las combinaciones entre ellos.

En un estudio sobre la diversidad genética de maíces nativos de México, se encontró que razas como el olotillo, tepecintle, tuxpeño, vandeño, dzitbacal y nal tel están agrupadas en un subgrupo al que se denominó maíces del Golfo de México, Pacífico Sur y Península de Yucatán (González et al., 2013). Una característica que diferencia a este subgrupo de las otras razas, es su mayor número de variabilidad alélica, misma que se atribuyó a la amplia distribución geográfica que presentan.

La principal característica que explica la subdivisión del grupo cuatro se atribuye a las diferencias en el ambiente donde se desarrollan las poblaciones. Los cambios que presentan los maíces de un mismo origen en ambientes diferentes, son posiblemente más intensos y dinámicos bajo las presiones que imponen las necesidades y circunstancias de los agricultores (Navarro-Garza et al., 2012). Esto puede explicar el porqué la variedad Tornamil se agrupó en un único subgrupo (4).

El subgrupo 1 lo integró únicamente al maíz pollito. Este maíz se alejó del resto de los maíces a excepción del chimbito. Esta similitud puede estar relacionada con que ambos materiales fueron colectados de la comunidad de Ricardo Flores Magón en parcelas cercanas, por lo que probablemente existe un intercambio de genes.
En el subgrupo 2 los materiales no estuvieron altamente relacionados entre sí, pero presentaron características particulares (probablemente relacionados al uso) que los hizo alejarse en un subgrupo aparte. El MNegro y el amarilloJOD, por sus colores intensos de granos, se han empleado únicamente para uso de alimentación familiar. Por otra parte, se considera que tienen sus orígenes del olotillo. Otro material (amarilloRFM) del mismo grupo, tiene como principal destino la alimentación animal; separado distancialmente de otros maíces por un potrero hacia un extremo e inclinaciones topográficas hacia el otro, aunado a una faja de árboles de vegetación secundaria. Estas características probablemente han propiciado la separación del flujo de genes, sin considerar las posibilidades de la distancia biológica (floración).

En el subgrupo 3 se concentraron la mayor parte de los maíces de la REBISE, en su gran mayoría materiales de generaciones avanzadas originados de variedades mejoradas de la raza tuxpeño, y en algunos casos con fuertes introgresiones de esta raza. En el subsubgrupo 1 se encontró una de las variedades mejoradas empleadas como testigo (Vs-424). Esta variedad fue introducida por la presidencia municipal. Sin embargo, no se adaptó a las condiciones y necesidades de los agricultores, y se dejó de sembrar. El subsubgrupo 2 se integró por el jarochoRFM y precozTP.

En el subsubgrupo 3 se encontró el maíz huesito, amarilloC, jarochoV, crema, amarilloV y morales. Estos materiales se han relacionado anteriormente con otras razas como olotillo y tepecintle. El subsubgrupo 4 integra al maíz chimbito, San Gregorio, olote rojo, jarochoTP, precozC y jarochoET; maíces que se caracterizan por tener fuerte influencia de la raza vandeño.

El análisis de coordenadas principales en un plano de dos dimensiones, reflejó una dispersión interracial de los materiales (Figura 4.). Hacia la parte negativa de la dimensión 2 (Dim-2) y de la dimensión 1 (Dim-1) se encontraron el híbrido H-MX3. Los maíces cónicos de Tlaxcala se ubicaron en el cuadrante negativo de la Dim-1 y positivo de la Dim-2. Mientras que la mayor parte de los maíces de la REBISE se direccionaron hacia la Dim-1; principalmente aquellos pertenecientes al subgrupo 3, descritos como variedades mejoradas y de generaciones avanzadas. En este sentido, mientras más se direccionen hacia la Dim-1 y negativa de la Dim-2, los maíces tienden a tener un mayor grado de mejoramiento o pertenecen a generaciones avanzadas. 


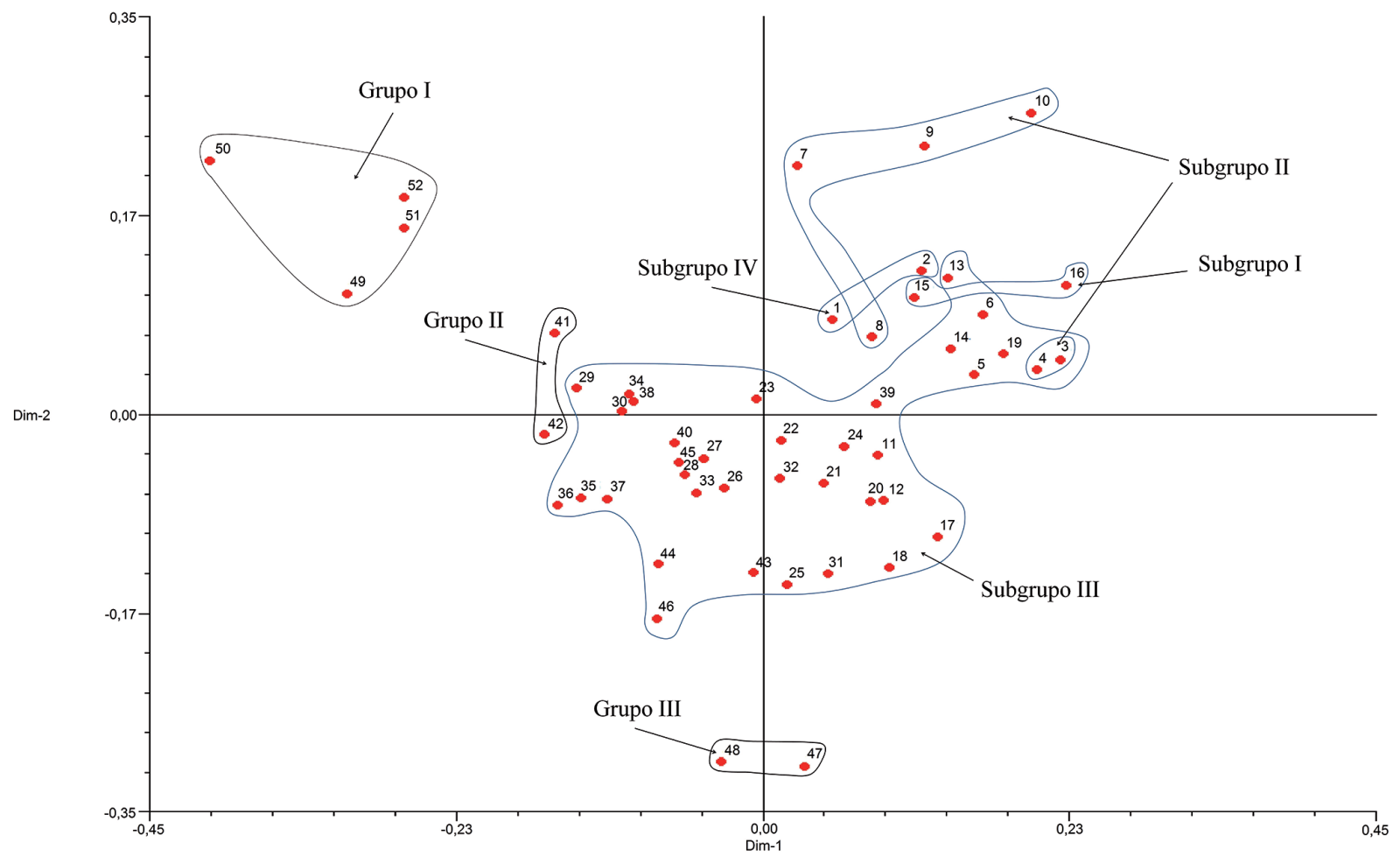

Figura 4. Distribución de los diecinueve maíces locales de la Reserva de la Biosfera La Sepultura (REBISE), Chiapas, México; en las dos primeras coordenadas principales, creado a partir de la distancia genética. México. 2013.

Figure 4. Distribution of nineteen local maize varieties from the Reserva de la Biosfera La Sepultura (REBISE), Chiapas, Mexico; in the first two main coordinates and created from the genetic distance. Mexico. 2013.

1) Tornamil; 2) Tornamil2; 3) MNegro; 4) MNegro2; 5) JarochoET; 6) JarochoET2; 7) AmarilloRFM; 8) AmarilloRFM2; 9) AmarilloJOD; 10) AmarilloJOD2; 11) PrecozC; 12) PrecozC2; 13) Chimbito; 14) Chimbito2; 15) Pollito; 16) Pollito2; 17) JarochoTP; 18) JarochoTP2; 19) Orojo; 20) ORojo2; 21) SGregorio; 22) SGregorio2; 23) JarochoRFM; 24) JarochoRFM2; 25) PrecozTP; 26) PrecozTP2; 27) Morales; 28) Morales2; 29) Crema; 30) Crema2; 31) AmarilloV; 32) AmarilloV2; 33) AmarilloC; 34) AmarilloC2; 35) Huesito; 36) Huesito2; 37) JarochoV; 38) JarochoV2; 39) Costeño1; 40) Costeño2; 41) Teocintle; 42) Teocintle2; 43) Vs-424-1; 44) Vs-424-2; 45) Vs-536-1; 46) Vs-536-2; 47) H-MX3-1; 48) H-MX3-2; 49) CónicoChalqueño; 50) CónicoChalqueño2; 51) ElotesCónicos1; 52) ElotesCónicos2.

Por el contrario, los maíces que se agrupan hacia la parte positiva de la Dim-2, presentan menor influencia de maíces mejorados y conservan sus características de origen.

En resumen, la relación que presentaron estos materiales como grupo 4, muestra la importancia que tiene la interacción de las diferentes razas en la formación de variedades locales y la selección del agricultor con base a su uso. Por lo tanto, una descripción lógica de estos materiales locales debe fundamentarse en el conjunto de respuestas posibles que se obtienen al interactuar más de una raza, y no necesariamente describirlas como la presencia de muchas razas en una población. Además, la relación que presentaron las poblaciones en el subgrupo 3 puede ser debidas a la considerable cantidad de flujo de genes entre poblaciones (maíces resultados de más de una raza), así como la fuerte selección divergente para características de interés antropogénicos (Canul, 2009). 


\section{AGRADECIMIENTOS}

Los autores agradecen a la Secretaría de Educación Pública (SEP) y al Consejo Nacional de Ciencia y Tecnología (CONACYT) por el financiamiento para el desarrollo de la presente investigación a través del proyecto: Caracterización socio-agronómica de maíces locales con potencial de uso múltiple en la Frailesca, Chiapas. Así también desean agradecer al Centro Internacional de Mejoramiento de Maíz y Trigo (CIMMYT) por el apoyo brindado para la realización de parte del trabajo de campo que culminó en la tesis del primer autor.

\section{LITERATURA CITADA}

Aguilar, J. 2010. Informe final del estudio técnico: Validación de semilla y del proceso de mantenimiento de agroecosistema en los ejidos de California, Nueva Esperanza y Flores Magón localizados en la zona de amortiguamiento de la reserva de la Biosfera la Sepultura. Municipio de Villaflores, Chiapas, MEX.

Akagi, H., Y. Yokozeki, A. Inagaki, A. Nakamura, and T. Fujimura. 1996. A codominant DNA marker closely linked to the rice nuclear restorer gene, $\mathrm{RF}-1$, identified with inter-SSR fingerprinting. Genome 39:1205-9.

Asemota, H.N. 1995. A fast, simple, and efficient miniscale method for the preparation of DNA from tissues of yam (Dioscorea spp.). Plant Mol. Biol. Rep. 13:214-218.

Bellon, M.R., and S.B. Brush. 1994. Keepers of maize in Chiapas, México. Econ. Bot. 48:196-209.

Berilli, A.P., M.G. Pereira, S.A. Gonçalves, K.S. da-Cunha, H.C.C. Ramos, G.A. Souza Filho, and A.T. doAmaral-Júnior. 2011. Use of molecular markers in reciprocal recurrent selection of maize increases heterosis effects. Genet. Mol. Res. 10:2589-2596.

Blair, M.W., O. Panaud, and S.R. Mccouch. 1999. Intersimple sequence repeat (ISSR) amplification for analysis of microsatellite motif frequency and fingerprinting in rice (Oryza sativa L.). Theor. Appl. Genet. 98:780-792.

Canul, K.J. 2009. Diversidad genética de poblaciones nativas de maíz y su mantenimiento en el sistema milpa de Yucatán. Tesis Doc. Colegio de Postgraduados, Texcoco, MEX.

Carvalho, V.P., P.M. Ruas, C.F. Ruas, J.M. Ferreira, and R.M. Moreira. 2002. Assessment of genetic diversity in maize (Zea mays L.) landraces using inter simple sequence repeat (ISSR) markers. Crop Breed. Appl. Biotechnol. 2:557-568.

Dice, L.R. 1945. Measures of the amount of ecologic association between species. Ecol. 26:297-302.

Do-Amaral-Júnior, A.T., E.C. de Olivera, G.L. Azeredo, C.A. Scapim, L.S. Candido, C.S. Rodriguez da, C. Vittorazzi, and C.K. Silva da. 2011. Assessment of genetic diversity among maize accessions using inter simple sequence repeats (ISSR) markers. Afr. J. Biotechnol. 10:15462-15469.

Durán-Hernández, D., G.F. Gutiérrez-Hernández, J.L. Arellano-Vázquez, E. García-Ramírez, y J. Virgen-Vargas. 2011. Caracterización molecular y germinación de semillas de maíces criollos azules con envejecimiento acelerado. Agron. Mesoam. 22:11-20.

González, C.M., R.N. Palacios, B.A. Espinoza, y S.C. Bedoya. 2013. Diversidad genética en maíces nativos mexicanos tropicales. Rev. Fitotec. Mex. 36:329-338.

Gutiérrez, G.F., E. García, J.L. Arellano, y J. Virgen. 2009. Comparación molecular de la identidad genética de genotipos de maíz de diferente edad. Agron. Mesoam. 20:01-09.

Hernández-Ramos, M.A. 2014. Maíces locales con potencial de uso múltiple en un área natural protegida de Chiapas. Tesis de M.Sc., Universidad Autónoma de Chiapas, Villaflores Chiapas, MEX.

Hufford, M.B., P. Lubinksy, T. Pyhäjärvi, M.T. Devengenzo, N.C. Ellstrand, and J. Ross-Ibarra. 2013. Correction: the genomic signature of crop-wild introgression in maize. PLoS Genet 9:e1003477.

INEGI (Instituto Nacional de Estadística, Geografía e Informática). 2010. México en el mundo. INEGI, MEX.

Joshi, S.P., V.S. Gupta, R.K. Aggarwal, P.K. Ranjekar, and D.S. Brar. 2000. Genetic diversity and phylogenetic relationship as revealed by inter simple sequence repeat (ISSR) polymorphism in the genus Oryza. Theor. Appl. Genet. 100:1311-1320.

Kato, T.A., C. Mapes, I.M. Mera, J.A. Serratos, y R.A. Bye. 2009. Origen y diversificación del maíz: una revisión analítica. Universidad Nacional Autónoma de México, Comisión Nacional para el Conocimiento y Uso de la Biodiversidad, MEX.

Keleman, A., J. Hellin, and M.R. Bellon. 2009. Maize diversity, rural development policy, and farmers' practices: lessons from Chiapas, Mexico. The Geographical J. 175:52-70.

Kumar, A., L. Arya, V. Kumar, and S. Sharma. 2006. Inter simple sequence repeat (ISSR) analysis of 
cytoplasmic male sterile, male fertile lines and hybrids of pearlmillet [Pennisetum glaucum (L.) R.Br.]. Indian J. Crop Sci. 1:117-119.

Legendre, L., and P. Legendre. 1983. Numerical ecology. $2^{\text {nd }}$ ed. Elsevier, Amsterdam, HOL.

Louette, D. 1996. Intercambio de semilla entre agricultores y flujo genético entre variedades de maíz en sistemas agrícolas tradicionales. En: J.A. Serratos et al., editores, Flujo genético entre maíz criollo, maíz mejorado y teocintle: implicaciones para el maíz transgénico. CIMMYT, MEX. p. 60-71.

Louette, D. 1999. Traditional management of seed and genetic diversity: what is a landrace? In: S.B. Brush, editor, Genes in the field: on farm conservation of crop. Lewis Publishers, Boca Ratón, FL, USA. p. 42-109.

Luo, S.G., B. Guanghong, and Y.S. Wu. 2011. Identification of ISSR marker for main maize hybrid cultivars and their parents in Xinjiang. Сейфуллина 67(1):131-135.

Mbuya, K., K.K. Nkongolo, R. Narendrula, A. KalonjiMbuyi, and R.V. Kizungu. 2012. Development of quality protein maize (QPM) inbred lines and genetic diversity assessed with ISSR marker in a maize breeding program. Am. J. Exp. Agric. 2:626-640.

McGregor, C.E., C.A. Lambert, M.M. Greyling, J.H. Louw, and L. Warnich. 2000. A comparative assesment of DNA fingerprinting techniques (RAPD, ISSR, AFLP and SSR) in tetraploid potato (Solanum tuberosum L.) germplasm. Euphytica 113:135-144.

Morales, Y.M.L. 2002. Caracterización genotípica de plantas de maíz (Zea mays L.) utilizando secuencias microsatélites distribuidas uniformemente sobre el genoma. Tesis Lic., Universidad de Belgrado, ARG.

Morgante, M., and A.M. Olivieri. 1993. PSR-ampliffied microsatellites as markers in plant genetic. Plant. J. 3:175-182.

Navarro-Garza, H., M. Hernández-Flores, F. CastilloGonzález, y M.A. Pérez-Olvera. 2012. Diversidad y caracterización de maíces criollos. Estudio de caso en sistemas de cultivo en la costa chica de Guerrero, México. Agricultura y Sociedad 9:149-165.

Ortega, C.A., H.M. Guerrero, A.O. Cota, y O.R. Preciado. 2011. Situación actual de los maíces nativos y sus parientes silvestres en México. En: R.E. Preciado, y S. Montes, editores, Amplitud, aprovechamiento y riesgos de la diversidad genética de maíz en México. Sociedad Mexicana de Fitogenética A.C., Chapingo, Edo., MEX. p. 25.

Ortiz, E., A. Carballo, A., Muñoz, y F. González. 2010. Efecto de la dispersión de polen en la producción de semilla de maíz, en Texcoco, México. Agron. Mesoam. 21:289-297.

Perales, H., y C. Hernández. 2005. Diversidad de maíz en Chiapas. En: M. González-Espinosa et al., editores, Diversidad biológica de Chiapas. Plaza y Valdés, ECOSUR, COCYTECH, México, DF, MEX. p. 337-355.

Pérez, M., P. Zirilli, N. Ulrich, L. Setten, y A. Escadón. 2010. Caracterización molecular en el género Mecardonia Ruiz \& Pav. (Plantaginaceae) utilizando marcadores ISSR. Rev. Fac. Agron. La Plata 109(1):23-30.

Pradeep, R., N. Sarla, and E.A. Siddiq. 2002. Inter simple sequence repeats (ISSR) polymorphism and its aplication in plant breeding. Euphytica 128(1):9-17.

PROMAC (Programa de Conservación Maíz Criollo). 2011. Lineamientos para otorgar el apoyo. PROMAC, SEMARNAT, MEX. http://www.conanp.gob.mx/ maiz_criollo/. (consultado 24 abr. 2015).

Ratnaparkne, M.B., D.K. Santra, A. Tullu, and F.J. Muehlbauer. 1998. Inheritance of intersimplesequence-repeat polymorphisms and linkage with a Fusarium wilt resistance gene in chickpea. Ther. Appl. Genet. 96:348-353.

Rentaría-Alcántara, M. 2007. Breve revisión de los marcadores moleculares. En: L.E. Eguiarte, et al., editores, Ecología molecular. SEMARNAT, MEX. p. 541-566.

Sneath, P.H., and R.R. Sokal. 1973. Numeric taxonomy: the principles and practice of numerical classification. W.H. Freemanadn Company, CA, USA.

Sokal, R.R., and C.D. Michener. 1958. A statistical method for evaluating systematic relationships. University of Kansas Scientific Bulletin, USA.

Solís, L.M. 2012. Caracterización molecular de accesiones de piñón (Jatropha curcas L.) del estado de Chiapas. Tesis M.Sc., Universidad Autónoma de Chiapas, Villaflores, Chiapas, MEX.

Taramino, G., and S. Tingey. 1996. Simple sequence repeats for germplasm analysis and mapping in maize. Genome 39:277-287.

Vásquez, C.M., G.B. Lorena, L.A. José, M.S. Fidel, y M.J. Castillo. 2003. Calidad de grano y tortilla de maíces criollos y sus retrocruzas. Rev. Fitotec. Mex. 26:231-238.

Velasco, P.A., T.J. Velasco, y T.A. Velasco. 2005. Estructura socioeconómica y política de Chiapas. $2^{\text {a }}$ ed. Talleres de Impresora Roma, Chiapas, MEX.

Wellhausen, E.J., L.M. Roberts, X.E. Hernández, y P.C. Mangelsdorf. 1951. Razas de maíz en México. Su origen, características y distribución. Folleto técnico No. 5. Secretaría de Agricultura y Ganadería, México, D.F., MEX. 
